\title{
Therapeutic responses and prognosis in Korean adult-onset Still's disease
}

\author{
Hyoun-Ah Kim*, Jeong-Mi An, Chang-Hee Suh \\ From 6th European Workshop on Immune-Mediated Inflammatory Diseases \\ Nice, France. 23-25 November 2011
}

\section{Background}

To date, the treatment of adult onset Still's disease (AOSD) has been largely empirical; therefore, this study was conducted to investigate the response to therapy and prognostic factors of AOSD. We describe the clinical features of 54 Korean patients with AOSD and provide an analysis of the therapeutic responses and prognostic factors associated with unfavorable outcomes and mortality in these cases.

\section{Method}

Fifty-four Korean patients with AOSD were enrolled based on Yamaguchi's criteria. We retrospectively analyzed the treatments and prognosis.

\section{Results}

Thirty-nine patients $(72.2 \%)$ were female and the average age at disease onset was 37.3 years. Twenty-nine patients had a monocyclic disease (53.7\%), five had a polycyclic (9.3\%) and fifteen had a chronic articular disease (27.7\%) and five died (9.3\%). The elevated ESR and corticosteroids refractoriness were associated with poor prognosis ( $p=0.023$ and $p=0.009$, respectively). The patients that died were older than those survived $(49.2 \pm 11.8$ vs $42.2 \pm 14$ year-old, $p=0.024)$. Forty-two patients were treated with nonsteroidal anti-inflammatory drugs; however, they also needed corticosteroids and intravenous immunoglobulin (IVIG). Among 50 patients treated with high-dose corticosteroids, 21 patients (42\%) were resistant to corticosteroids and treated with IVIG or anti-tumor necrosis factor (TNF) agents. Of the 23 patients medicated with IVIG, the prognosis was better in IVIG-responsive patients, indicating a therapeutic effect. Methotrexate was the most commonly used disease modifying antirheumatic drugs

Dept. of Rheumatology, Ajou University School of Medicine, Suwon, Republic of Korea
(27 patients, 50\%) and the corticosteroid requirements were lower in the methotrexate-responsive patients.

\section{Conclusion}

Approximately a half of Korean AOSD patients had a poor prognosis including polycyclic or chronic articular disease, or mortality. In addition, there was no clinical response to NSAIDs and $42 \%$ of patients were corticosteroids resistant and therefore required IVIG and anti-TNF agents. Methotrexate showed a corticosteroid-sparing effect. The elevated serum ESR and non-response to corticosteroids were significantly associated with poor prognosis. Finally, patients who died were older than those who survived.

Published: 23 November 2011

doi:10.1186/1479-5876-9-S2-P35

Cite this article as: Kim et al:: Therapeutic responses and prognosis in Korean adult-onset Still's disease. Journal of Translational Medicine 20119 (Suppl 2):P35.

Submit your next manuscript to BioMed Central and take full advantage of:

- Convenient online submission

- Thorough peer review

- No space constraints or color figure charges

- Immediate publication on acceptance

- Inclusion in PubMed, CAS, Scopus and Google Scholar

- Research which is freely available for redistribution 